\title{
Probing pseudo-Goldstone dark matter at the LHC
}

\author{
Katri Huitu, ${ }^{*}$ Niko Koivunen, ${ }^{\dagger}$ Oleg Lebedev, ${ }^{\star}$ and Subhadeep Mondal $^{\S}$ \\ Department of Physics, and Helsinki Institute of Physics, University of Helsinki, \\ P.O. Box 64, Helsinki FI-00014, Finland \\ Takashi Tomall \\ Department of Physics, Kyoto University, Kyoto 606-8502, Japan
}

(Received 16 April 2019; published 9 July 2019)

\begin{abstract}
Pseudo-Goldstone dark matter coupled to the Standard Model via the Higgs portal offers an attractive framework for phenomenologically viable pseudoscalar dark matter. It enjoys natural suppression of the direct detection rate due to the vanishing of the relevant (tree level) Goldstone boson vertex at zero momentum transfer, which makes light WIMP-like dark matter consistent with the strong current bounds. In this work, we explore prospects of detecting pseudo-Goldstone dark matter at the LHC, focusing on the vector boson fusion channel with missing energy. We find that, in substantial regions of parameter space, relatively light dark matter $\left(m_{\chi}<150 \mathrm{GeV}\right)$ can be discovered in the high luminosity run as long as it is produced in decays of the Higgs-like bosons.
\end{abstract}

DOI: 10.1103/PhysRevD.100.015009

\section{INTRODUCTION}

The dark matter (DM) puzzle remains one of the pressing issues in modern physics. Various particle physics models have been constructed which fit known properties of dark matter. Among these, the weakly interacting massive particle (WIMP) paradigm remains one of the frontrunners.

Recently, the electroweak-scale dark matter models have come under increasing pressure from direct detection experiments which have so far found null results $[1,2]$. These constrain the nucleon-dark matter interactions at (effectively) zero momentum transfer. An interesting option to evade such bounds is to employ the property of Goldstone bosons that the relevant vertices vanish at zero momentum transfer (Fig. 1), while they are otherwise unsuppressed [3]. It is important that this statement also applies to massive pseudoGoldstone bosons, which allows one to use this mechanism to suppress direct detection rates of WIMP-like dark matter. In this case, the nucleon-dark matter interaction arises at one loop level and satisfies the XENON1T bound naturally.

*katri.huitu@ helsinki.fi

niko.koivunen@helsinki.fi

‡oleg.lebedev@helsinki.fi

subhadeep.mondal@helsinki.fi

"takashi.toma@gauge.scphys.kyoto-u.ac.jp

Published by the American Physical Society under the terms of the Creative Commons Attribution 4.0 International license. Further distribution of this work must maintain attribution to the author(s) and the published article's title, journal citation, and DOI. Funded by SCOAP ${ }^{3}$.
In this work, we explore the prospects of probing pseudoGoldstone $\mathrm{DM}^{1}$ at the LHC. A promising channel with a relatively low background is vector boson fusion (VBF) Higgs production followed by its invisible decay into DM pairs [15]. The minimal model contains two Higgs-like scalars whose invisible decays contribute to this missing $E_{T}$ signature. Since pseudo-Goldstone bosons are naturally light, these channels are expected to be allowed kinematically. The relevant constraints that restrict the efficiency of DM production are due to the current bounds on the Higgs invisible decay, heavy Higgs searches, and indirect DM detection. We find that despite the strong constraints, light DM can be probed efficiently in the high luminosity run of the LHC.

\section{OVERVIEW OF THE MODEL AND EXISTING CONSTRAINTS}

Consider a simple extension of the Standard Model with a complex scalar field $S$ carrying a global U(1) charge [3]. The most general renormalizable scalar potential invariant under global U(1) transformations $S \rightarrow e^{i \alpha} S$ is

$$
\begin{aligned}
V_{0}= & -\frac{\mu_{H}^{2}}{2} H^{\dagger} H-\frac{\mu_{S}^{2}}{2} S^{*} S+\frac{\lambda_{H}}{2}\left(H^{\dagger} H\right)^{2}+\lambda_{H S} H^{\dagger} H S^{*} S \\
& +\frac{\lambda_{S}}{2}\left(S^{*} S\right)^{2} .
\end{aligned}
$$

\footnotetext{
${ }^{1}$ Pseudo-Goldstone DM in a different context has been considered in [4-14].
} 


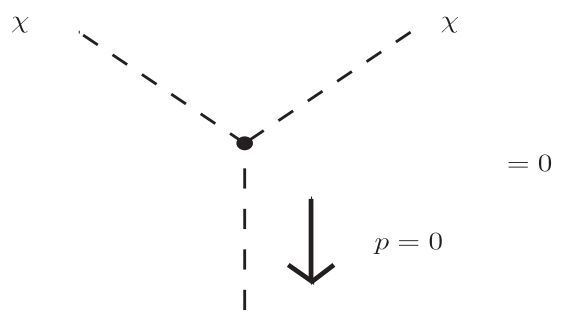

FIG. 1. The vertex involving a (pseudo-)Goldstone boson $\chi$ on shell and a scalar vanishes at zero momentum transfer. This persists for a massive $\chi$ as well [3].

The U(1) gets broken spontaneously when $S$ acquires a vacuum expectation value (VEV). This would result in the presence of a massless Goldstone boson. To avoid it, we introduce a soft breaking mass term for $S$ :

$$
V_{\text {soft }}=-\frac{\mu_{S}^{\prime 2}}{4} S^{2}+\text { H.c. }
$$

with the full scalar potential being

$$
V=V_{0}+V_{\text {soft }}
$$

In this case, a nonzero $S$ VEV generates a pseudoGoldstone boson with mass $\mu_{S}^{\prime}$.

The parameter $\mu_{S}^{\prime 2}$ can always be made real and positive by phase redefinition. The scalar potential $V$ is therefore invariant under " $\mathrm{CP}$ symmetry"

$$
S \rightarrow S^{*}
$$

It is easy to show that $S$ develops a real VEV such that this symmetry is unbroken by the vacuum [3]. As a result, $\operatorname{Im} S$ couples to other fields in pairs and is therefore stable.

The scalar fields are parametrized as

$$
H=\frac{1}{\sqrt{2}}\left(\begin{array}{c}
0 \\
v+h
\end{array}\right)
$$

and

$$
S=\frac{1}{\sqrt{2}}\left(v_{s}+s+i \chi\right) .
$$

The stability of the pseudoscalar $\chi$ is guaranteed by the $C P$ symmetry and it will play the role of pseudo-Goldstone dark matter in our model.

The potential minimization conditions read

$$
\begin{gathered}
\mu_{H}^{2}=\lambda_{H} v^{2}+\lambda_{H S} v_{s}^{2}, \\
\mu_{S}^{2}=\lambda_{H S} v^{2}+\lambda_{S} v_{s}^{2}-\mu_{S}^{\prime 2} .
\end{gathered}
$$

Using these relations the parameters $\mu_{H}^{2}$ and $\mu_{S}^{2}$ can be eliminated from the scalar potential. The $C P$-even scalars $h$ and $s$ mix due to the presence of the portal coupling $\lambda_{H S}$ in the potential and the mass matrix in the $(h, s)$ basis is

$$
\mathcal{M}^{2}=\left(\begin{array}{cc}
\lambda_{H} v^{2} & \lambda_{H S} v v_{s} \\
\lambda_{H S} v v_{s} & \lambda_{S} v_{s}^{2}
\end{array}\right)
$$

It is diagonalized by the orthogonal transformation

$$
O^{T} \mathcal{M}^{2} O=\left(\begin{array}{cc}
m_{h_{1}}^{2} & 0 \\
0 & m_{h_{2}}^{2}
\end{array}\right),
$$

with

$$
O=\left(\begin{array}{cc}
\cos \theta & \sin \theta \\
-\sin \theta & \cos \theta
\end{array}\right)
$$

where $\theta$ is the mixing angle,

$$
\tan 2 \theta=\frac{2 \lambda_{H S} v v_{s}}{\lambda_{S} v_{s}^{2}-\lambda_{H} v^{2}} .
$$

The eigenvalues of the mass-squared matrix are

$$
m_{h_{1}, h_{2}}^{2}=\frac{1}{2}\left(\lambda_{H} v^{2}+\lambda_{S} v_{s}^{2} \mp \frac{\lambda_{s} v_{s}^{2}-\lambda_{H} v^{2}}{\cos 2 \theta}\right) .
$$

Here $h_{1}$ is identified with the SM-like Higgs boson observed at the LHC. The pseudoscalar mass is given by the soft mass term:

$$
m_{\chi}^{2}=\mu_{S}^{\prime 2}
$$

The six input parameters of the scalar potential are subject to two experimental constraints: $v=246 \mathrm{GeV}$ and $m_{h_{1}}=125 \mathrm{GeV}$, leaving four free parameters which we choose as $m_{h_{2}}, \theta, v_{s}$, and $m_{\chi}$.

We note that a number of variations of the Higgs portal model with a complex scalar singlet have been considered in the literature [16-20].

\section{A. U(1) breaking couplings as spurions}

Here we provide a rationale for our choice of the U(1) breaking couplings following [3]. The main feature is that the odd powers of $S$ are absent and the higher even powers of $S$ are suppressed. This can be justified by treating our setup as the low energy limit of a more fundamental theory containing additional states at a high energy scale.

The simplest option would be to introduce a heavy complex singlet $\Phi$ which carries an even U(1) charge $q_{\Phi}$, while assigning $S$ an odd charge $q_{S}$. The couplings allowed by $\mathrm{U}(1)$ symmetry involve even powers of $S$, e.g.,

$$
\frac{S^{2 k} \Phi^{l}}{\Lambda^{2 k+l-4}},
$$


where $k, l$ are integer and $\Lambda$ is some high energy scale. The (gauged) U(1) is broken at the high scale by a VEV of $\Phi$ to a $Z_{2}$ subgroup which acts on $S$ as $S \rightarrow-S$. Define

$$
n \equiv-2 \frac{q_{S}}{q_{\Phi}}, \quad \epsilon \equiv \frac{\langle\Phi\rangle}{\Lambda},
$$

where $\epsilon \ll 1$. We then find

$\mu_{S}^{\prime 2} \sim\langle\Phi\rangle^{2} \epsilon^{n-2}, \quad \lambda_{H S}^{\prime} \sim \lambda_{S}^{\prime \prime} \sim \epsilon^{n}, \quad \lambda_{S}^{\prime} \sim \epsilon^{2 n}$,

where $\lambda_{H S}^{\prime}, \lambda_{S}^{\prime \prime}, \lambda_{S}^{\prime}$ are the coefficients of the $|H|^{2} S^{2},|S|^{2} S^{2}, S^{4}$ terms in the scalar potential, respectively. Clearly, for small $\epsilon$, $\mu_{S}^{\prime 2}$ is the leading term, while the others are highly suppressed. We thus recover our effective setup as the low energy limit.

In this example, one may require $C P$ symmetry, in which case dark matter stability is automatic and enforced by $S \rightarrow S^{*}$. If $C P$ is violated, dark matter is unstable and its decay is induced by the complex phases in the higherdimension operators. For $\epsilon \ll 1$, its lifetime can be made much longer than the age of the Universe [3], such that it can be treated as stable for all practical purposes.

We thus conclude that our assumptions can easily be justified by treating the $\mathrm{U}(1)$ breaking couplings as spurions.

\section{B. Cancellation of the direct DM detection amplitude}

The main feature of our dark matter framework is that the direct detection amplitude vanishes at tree level and zero momentum transfer [3]. The leading DM-nucleon scattering process is shown in Fig. 2. Let us examine how the cancellation comes about in the mass eigenstate basis.

The relevant interaction terms are given by

$$
\begin{aligned}
& \mathcal{L} \supset \frac{1}{2 v_{s}} \chi^{2}\left(h_{1} m_{h_{1}}^{2} \sin \theta-h_{2} m_{h_{2}}^{2} \cos \theta\right), \\
& \mathcal{L} \supset-\left(h_{1} \cos \theta+h_{2} \sin \theta\right) \sum_{f} \frac{m_{f}}{v} \bar{f} f,
\end{aligned}
$$

where $f$ denotes the SM fermions. Thus, the tree-level direct-detection scattering amplitude is given by

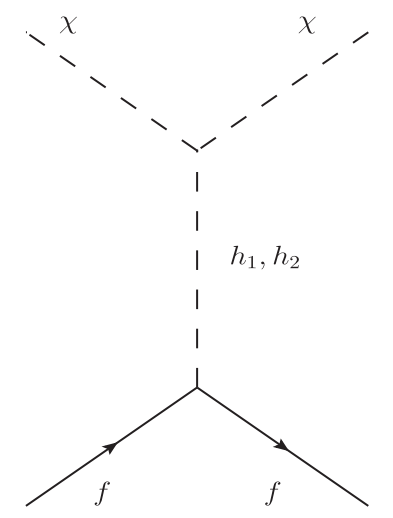

FIG. 2. DM-nucleon scattering at tree level.

$$
\begin{aligned}
\mathcal{A}_{d d}(t) & \propto \sin \theta \cos \theta\left(\frac{m_{h_{2}}^{2}}{t-m_{h_{2}}^{2}}-\frac{m_{h_{1}}^{2}}{t-m_{h_{1}}^{2}}\right) \\
& \simeq \sin \theta \cos \theta \frac{t\left(m_{h_{2}}^{2}-m_{h_{1}}^{2}\right)}{m_{h_{1}}^{2} m_{h_{2}}^{2}} \simeq 0
\end{aligned}
$$

since the momentum transfer in this process is negligibly small, $t \simeq 0$. Thus, the contributions from $h_{1}$ and $h_{2}$ exchange cancel each other up to tiny corrections of order $t /(100 \mathrm{GeV})^{2}$. This cancellation does not require any special relation between $m_{h_{1}}$ and $m_{h_{2}}$ and occurs for any parameter choice. It is of course a result of the pseudoGoldstone nature of our dark matter. In terms of the polar coordinates, $S=\rho e^{i \phi}$, where $\phi$ is identified with dark matter, one finds that the $\phi \phi \rho$ vertex vanishes for $\phi$ on shell and zero momentum of $\rho$. This statement is specific to the explicit U(1) breaking by a mass term $S^{2}$ and does not hold for higher dimensional operators.

The cancellation is spoiled by loop corrections which generate U(1) breaking terms of dimension 4 , e.g., $S^{4}$. The resulting direct detection cross section is in the ballpark of $10^{-49} \mathrm{~cm}^{2}$ [3], which is significantly below the current bounds. A detailed analysis of these loop corrections has recently been performed in $[21,22]$.

In our framework, the observed DM relic density can have both thermal and nonthermal origin. The DM annihilation cross section does not suffer from the above cancellation since the momentum transfer is large in this case. Thus, the correct relic abundance can be achieved through the usual WIMP annihilation mechanism [3]. In this work however, we consider a more general possibility that the DM production mechanism may be nonthermal, which allows for a wider range of DM masses including $m_{\chi}$ as low as $10 \mathrm{GeV}$.

\section{Invisible decay branching ratio}

In this study, we aim to probe the invisible decay of the $C P$-even Higgses $h_{1}$ and $h_{2}$ into a pair of DM particles $\chi$. When such decays are allowed, the VBF Higgs production with missing energy provides a promising channel for dark matter detection. The $\chi-\chi-h_{1,2}$ couplings are given by

$$
\begin{aligned}
\kappa_{\chi \chi h_{1}} & =\frac{m_{h_{1}}^{2}}{2 v_{s}} \sin \theta, \\
\kappa_{\chi \chi h_{2}} & =-\frac{m_{h_{2}}^{2}}{2 v_{s}} \cos \theta
\end{aligned}
$$

leading to the invisible decay widths

$$
\begin{gathered}
\Gamma\left(h_{1} \rightarrow \chi \chi\right)=\frac{m_{h_{1}}^{3} \sin ^{2} \theta \sqrt{1-4 \frac{m_{\chi}^{2}}{m_{h_{1}}^{2}}}}{32 \pi v_{s}^{2}}, \\
\Gamma\left(h_{2} \rightarrow \chi \chi\right)=\frac{m_{h_{2}}^{3} \cos ^{2} \theta \sqrt{1-4 \frac{m_{\chi}^{2}}{m_{h_{2}}^{2}}} .}{32 \pi v_{s}^{2}} .
\end{gathered}
$$



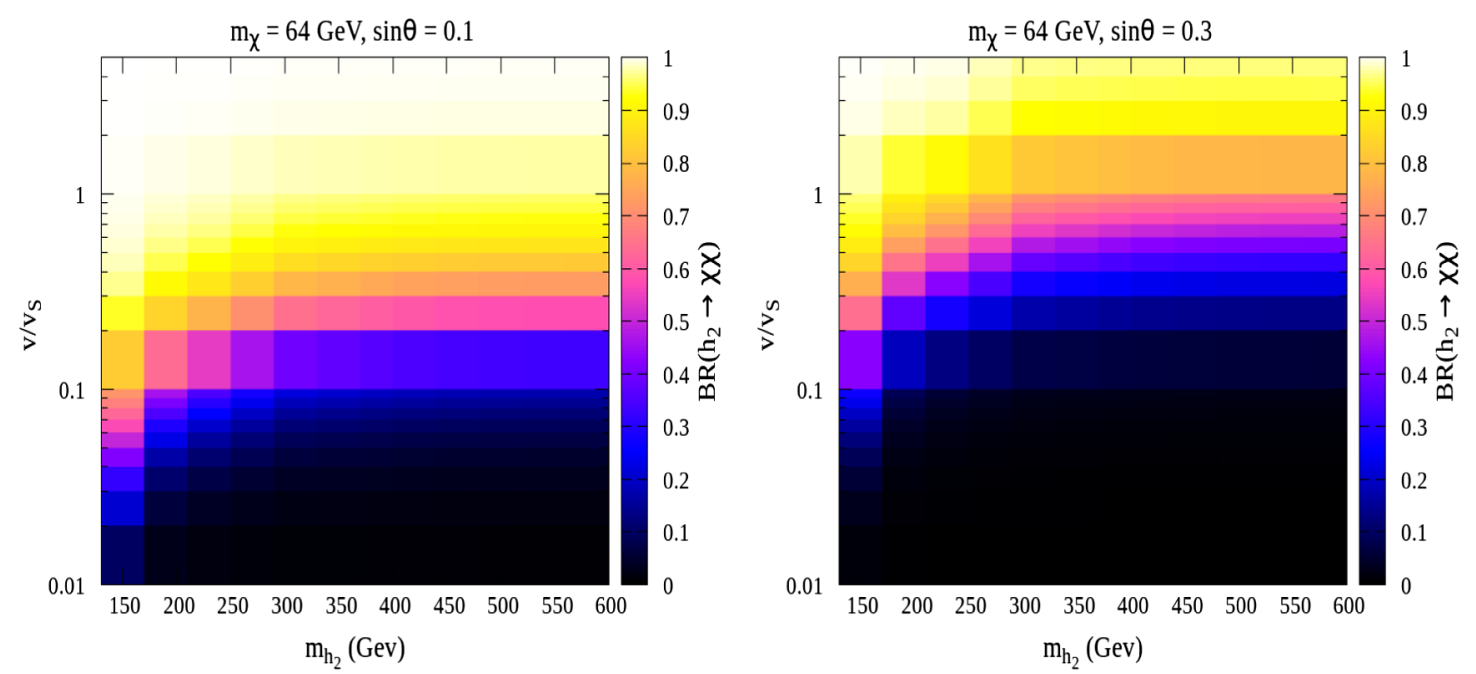

FIG. 3. Variation of $\operatorname{BR}\left(h_{2} \rightarrow \chi \chi\right)$ in the $\left(m_{h_{2}}, v / v_{s}\right)$ plane for representative parameter values.

The decay $h_{1} \rightarrow \chi \chi$ is quite constrained by the LHC Higgs data, with $\operatorname{BR}\left(h_{1} \rightarrow \chi \chi\right)$ not exceeding $10 \%$ as required by the Higgs signal strength observations [23]. On the other hand, the decay $h_{2} \rightarrow \chi \chi$ can be very efficient. It is important to include both of these DM production channels since either of them can dominate depending on the parameter choice.

Equation (2.21) shows that the DM couplings to the $C P$-even scalars grow with $m_{h_{1,2}}$ and $1 / v_{s}$. Given that $\sin \theta \lesssim 0.3$ as required by the $\left(h_{1}\right)$ Higgs coupling measurements [24], one concludes that $h_{2}$ couples to DM significantly stronger than $h_{1}$ does. For $h_{2}$, the competing decay modes are the $\sin \theta$-suppressed $h_{2} \rightarrow V V^{(*)}$ and, for larger $m_{h_{2}}, h_{2} \rightarrow h_{1} h_{1}$ and $h_{2} \rightarrow t \bar{t}$. The variation of $\operatorname{BR}\left(h_{2} \rightarrow \chi \chi\right)$ for typical parameter choices is shown in Fig. 3. We keep $m_{h_{2}}$ below $600 \mathrm{GeV}$ to have a substantial $h_{2}$-production cross section and choose $m_{\chi}=64 \mathrm{GeV}$ to evade the $\operatorname{BR}\left(h_{1} \rightarrow \chi \chi\right)$ constraint. We see that for $v / v_{s}>0.1$, the invisible decay mode is significant and often dominant.

\section{Constraints}

The model parameters $m_{h_{2}}, m_{\chi}, v_{s}$, and $\theta$ are constrained by various experiments. Perturbative unitarity considerations exclude small values of $v_{s} \ll v$, which for fixed scalar masses imply large quartic couplings. The mixing angle $\theta$ and dark matter mass $m_{\chi}$ are constrained by the LHC Higgs coupling data. In addition, depending on the choice of $\theta$, the heavy $C P$-even Higgs boson mass $m_{h_{2}}$ is subject to the LHC direct search bound. ${ }^{2}$ While the direct DM detection constraint is weak and superseded by that from perturbative unitarity in the relevant parameter range, the indirect DM

\footnotetext{
${ }^{2}$ A summary of analogous constraints in the real scalar extension of the SM can be found in [25].
}

detection constraint from the Fermi satellite is significant for relatively light DM. Finally, when $\chi$ is assumed to have been produced thermally, there is a PLANCK constraint on the DM annihilation cross section, which requires substantial DM-scalar couplings away from the resonance regions. The dark matter computations were performed using MICROMEGAs [26].

Below we delineate parameter space consistent with all of the constraints, keeping the spectrum at the electroweak scale and the mixing angle below 0.3.

\section{Constraints from unitarity, invisible Higgs decay and dark matter detection experiments}

Figure 4 shows the results of our numerical analysis at fixed representative values of $\sin \theta$ and $m_{h_{2}}$. The grey, purple, and orange regions are excluded by the perturbative unitarity constraint $\lambda_{S}<8 \pi / 3$ [27], the Higgs invisible decay bound [23], and the gamma-ray observations from dwarf spheroidal galaxies (dSphs) with 6 years and $15 \mathrm{dSphs}$ data by the Fermi-LAT Collaboration [28,29], respectively.

To constrain the invisible $h_{1}$ Higgs decay, we use the Higgs signal strength value $\mu=1.09_{-0.10}^{+0.11}$ obtained with $7+8 \mathrm{TeV}$ LHC data [23]. In our model, the effective $\mu$ is given by

$$
\mu=\cos ^{2} \theta\left(1-\mathrm{BR}_{\mathrm{inv}}\right),
$$

where $\mathrm{BR}_{\text {inv }}$ is the $h_{1}$ invisible decay branching ratio. The bound on $\mathrm{BR}_{\mathrm{inv}}$ is $\theta$ dependent: at $\sin \theta=0.1$, it gives $\mathrm{BR}_{\text {inv }}<10 \%$ at $2 \sigma$, while for $\sin \theta=0.3$ it strengthens to $\mathrm{BR}_{\mathrm{inv}}<2 \%$. The direct $13 \mathrm{TeV}$ bound $\mathrm{BR}_{\mathrm{inv}}<26 \%$ [24,30] is weaker, while the $13 \mathrm{TeV}$ constraints on the Higgs signal strength $1.13_{-0.08}^{+0.09}$ (ATLAS) [30] and $1.17 \pm$ 0.10 (CMS) [24] are only consistent with the SM at $2 \sigma$ level. It is therefore reasonable to use a conservative bound quoted above. 

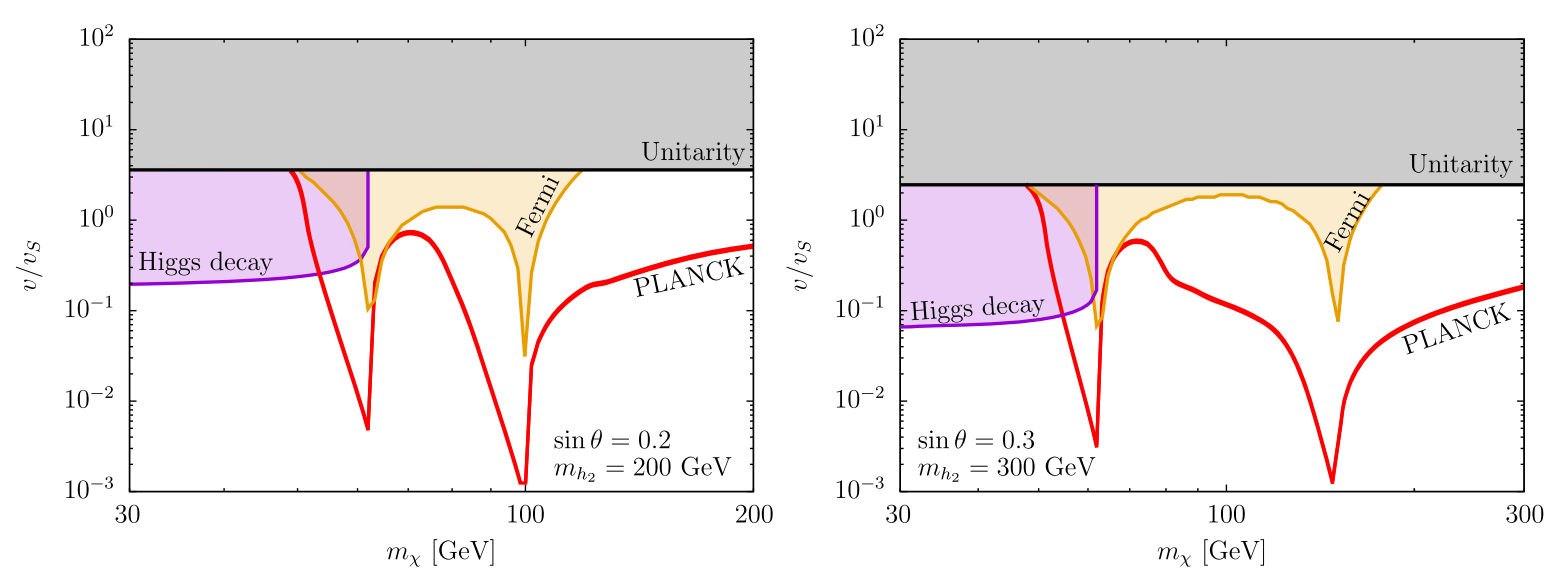

FIG. 4. $\left(m_{\chi}, v / v_{s}\right)$ parameter space. The colored regions are excluded by perturbative unitarity (grey), the Higgs invisible decay (purple), and gamma-ray observations (orange). The red band gives the correct thermal DM relic abundance according to PLANCK.

For the gamma-ray constraint, a Navarro-Frenk-White (NFW) dark matter profile is assumed [31]. The red band shows the allowed parameter range for thermal dark matter, whose abundance lies within a $3 \sigma$ interval of $\Omega_{\chi} h^{2}=0.1197 \pm 0.0022$ as reported by the PLANCK Collaboration [32]. One observes the presence of the characteristic dips associated with the resonant DM annihilation through $h_{1}$ and $h_{2}$. The DM couplings are allowed to be very small around these dips. The low mass end of the red bands, $m_{\chi}<m_{h_{1}} / 2$, is excluded by the invisible Higgs decay constraint, while the Fermi bound does not significantly affect the allowed parameter space for thermal DM. The direct DM detection constraint is loose and superseded by that from perturbative unitarity (grey area) [3].

The uncolored regions are allowed by all of the constraints as long as the DM production mechanism is nonthermal. In particular, $m_{\chi}=\mathcal{O}(10) \mathrm{GeV}$ is consistent with the Higgs invisible decay bound for small DM-Higgs couplings.

\section{Constraints from the LHC direct search}

Further constraints are imposed by the direct LHC search for Higgs-like states in various channels. We have taken into account the following $13 \mathrm{TeV}$ LHC results:

(i) The $h_{2} \rightarrow \gamma \gamma$ searches by ATLAS [33] and CMS [34]. The ATLAS search probes the heavy Higgs masses above $200 \mathrm{GeV}$, whereas the CMS lower bound is $500 \mathrm{GeV}$.

(ii) Searches for $h_{2} \rightarrow W W$ and $h_{2} \rightarrow Z Z$ in ATLAS [35-37] and CMS [38]. The CMS $h_{2} \rightarrow Z Z$ search
[38] probes the lowest mass range: $m_{h_{2}} \geq 130 \mathrm{GeV}$. The ATLAS $h_{2} \rightarrow W W$ and $h_{2} \rightarrow Z Z$ searches are sensitive to masses above $200 \mathrm{GeV}$. The combined ATLAS limits from $h_{2} \rightarrow W W$ and $h_{2} \rightarrow Z Z$ searches impose a limit on $m_{h_{2}} \geq 300 \mathrm{GeV}$.

(iii) The ATLAS searches for $h_{2} \rightarrow h_{1} h_{1}$ in 4b [39], $2 \gamma 2 b$ [40] and $2 W 2 \gamma$ final states, and the CMS searches in $2 \gamma 2 b$ [41] and $4 b$ [42] final states. The ATLAS searches and the CMS $4 b$ search probe the heavy Higgs masses above $260 \mathrm{GeV}$ and the CMS $2 \gamma 2 b$ search probes those above $250 \mathrm{GeV}$.

(iv) The ATLAS search for the invisible $h_{2}$ decay in the VBF production channel [43]. This probes the heavy Higgs mass range starting from $100 \mathrm{GeV}$.

We are interested in the low mass range $150-300 \mathrm{GeV}$ as this leads to the strongest signal. The above searches set a bound on the production cross section of the final state in question. In the narrow width approximation (NWA), it is given by

$$
\sigma_{\text {prod }}=\sigma\left(p p \rightarrow h_{2}\right) \times \mathrm{BR}\left(h_{2} \rightarrow \mathrm{SM}\right) .
$$

The $h_{2}$ production cross section is proportional to $\sin ^{2} \theta$,

$$
\sigma\left(p p \rightarrow h_{2}\right)=\sin ^{2} \theta \times \sigma_{p p \rightarrow h}^{\mathrm{SM}}\left(m_{h}=m_{h_{2}}\right),
$$

where $\sigma_{p p \rightarrow h}^{\mathrm{SM}}$ is the production cross section of the SM Higgs boson. The branching ratio for the $h_{2}$ decay to a given SM final state is

$$
\mathrm{BR}\left(h_{2} \rightarrow \mathrm{SM}\right)=\frac{\sin ^{2} \theta \Gamma_{h \rightarrow \mathrm{SM}}\left(m_{h}=m_{h_{2}}\right)}{\sin ^{2} \theta \Gamma_{h}^{\mathrm{tot}}\left(m_{h}=m_{h_{2}}\right)+\Gamma\left(h_{2} \rightarrow \chi \chi\right)+\Gamma\left(h_{2} \rightarrow h_{1} h_{1}\right)},
$$

where $\Gamma_{h \rightarrow \mathrm{SM}}$ is the SM Higgs decay rate to the final state and $\Gamma_{h}^{\mathrm{tot}}$ is the SM Higgs total decay width. 

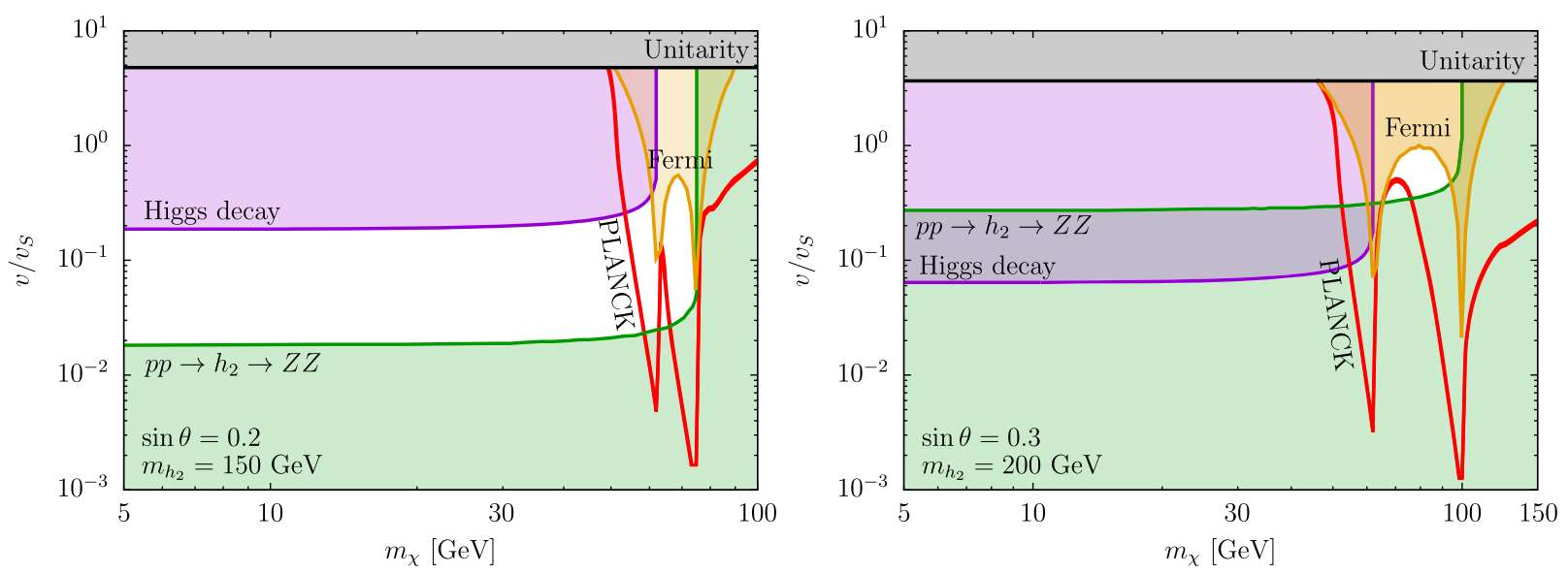

FIG. 5. LHC $h_{2}$-search constraints (green) in the $\left(m_{\chi}, v / v_{s}\right)$ plane. The other constraints are as in Fig. 4.

It is clear that $\sigma_{\text {prod }}$ receives two suppression factors: $\sin ^{2} \theta$ and the presence of nonstandard decay channels. For the mixing angle values $\sin \theta=0.1,0.2$, and 0.3 , the cross section $\sigma_{\text {prod }}$ is below the limit for the $\gamma \gamma, W W$, and $h_{1} h_{1}$ final states. The ZZ searches in both CMS [38] and ATLAS [37] and the combined $W W, Z Z$ search in ATLAS [36] are more sensitive and for $\sin \theta=0.2,0.3$ impose a nontrivial constraint shown in Fig. 5.

Below the $h_{2} \rightarrow h_{1} h_{1}$ kinematic threshold, the necessary suppression is provided by the invisible decay channel $h_{2} \rightarrow \chi \chi$ as long as $m_{h_{2}}>2 m_{\chi}$. While the $\sin \theta=0.1$ case is safe regardless of the efficiency of the invisible decay, at $\sin \theta=0.2,0.3$ a large $\mathrm{BR}_{\text {inv }}\left(h_{2}\right)$ is required in order for a light $h_{2}$ to be consistent with the LHC data. This excludes low values of $v / v_{s}$ at which the $\chi-\chi-h_{2}$ coupling is suppressed, as shown in Fig. 5. The constraint is rather sensitive to the exact value of $m_{h_{2}}$ : while the observed limit for $m_{h_{2}}=150 \mathrm{GeV}$ is lower than the corresponding SM value by a factor of 31 , the analogous factor for $m_{h_{2}}=$ $200 \mathrm{GeV}$ is only 22 . That means, in the latter case the $\sin ^{2} \theta=(0.2)^{2}$ suppression is enough to satisfy the bound at any $v_{s}$, while in the former case an efficient invisible decay channel is necessary.

It is interesting that at higher $m_{h_{2}} \sim 300 \mathrm{GeV}$, the above constraint disappears due to the additional decay channel $h_{2} \rightarrow h_{1} h_{1}$. The corresponding coupling

$C_{h_{1} h_{1} h_{2}}=-\frac{2 m_{h_{1}}^{2}+m_{h_{2}}^{2}}{4 v v_{s}} \sin 2 \theta\left(v_{s} \cos \theta+v \sin \theta\right)$

is quite large and remains unsuppressed at large $v_{s}$ making the standard Higgs decay channels much less efficient. Thus, both panels of Fig. 4 are consistent with the $h_{2}$ constraint.

\section{COLLIDER ANALYSIS}

We aim to probe our DM model via the missing energy signature in the VBF Higgs production channel (Fig. 6). There are of course other options as well. For example, Ref. [44] has explored gluon fusion with the initial or final

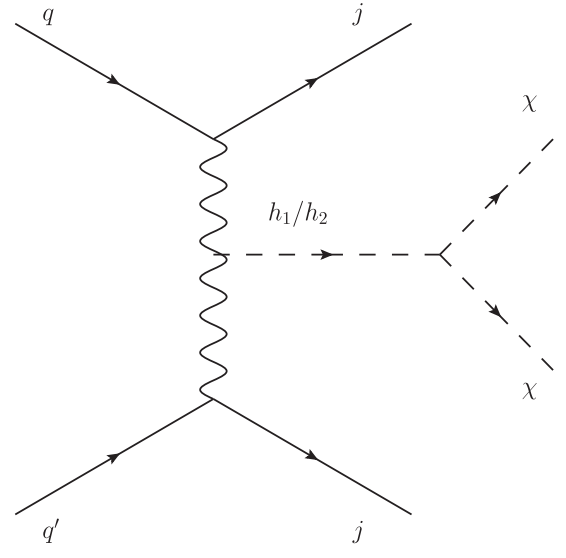

FIG. 6. DM pair production via vector boson fusion $(\mathrm{VBF})$ at the LHC.

state radiation accompanied by missing energy, within a somewhat different Higgs portal DM model. The problematic aspect in this case is the large QCD background. For the VBF mode, the background is lower since the jets produced in this process are forward and easier to tag on. Thus it appears to be a promising channel. ${ }^{3}$

We find that the process is efficient only when the DM pair is produced by on-shell $h_{1}$ and/or $h_{2}$ decays. Thus we will focus on the region $m_{h_{2}}>2 m_{\chi}$. In addition, due to the $\sin ^{2} \theta$ suppression, the heavy Higgs production cross section is significant only for the electroweak range masses. Although the $h_{1}$ production is unsuppressed by the mixing angle, the kinematic reach of $h_{1} \rightarrow \chi \chi$ is smaller than that for the $h_{2}$ decay and, furthermore, its branching ratio is already strongly constrained. As we detail below, both $h_{1}$

\footnotetext{
${ }^{3}$ In order to ascertain the fact that a VBF production channel is more sensitive compared to a gluon fusion production resulting in a monojet signal, we made an estimate with reference to the results in [44]. We found that with similar parameter choices, the significance factor in the VBF production channel can be larger by a factor $\sim 1.6$.
} 
and $h_{2}$ can give a dominant contribution to DM pair production, depending on the parameter region.

\section{A. Simulation details}

The ATLAS and CMS collaborations have explored the possibility of detecting invisible decay of the Higgs boson in the VBF channel and have optimized the corresponding cuts $[43,45]$. The definitions of the signal region and kinematic cuts are very similar in both of the analyses. In this work, we have adopted the CMS analysis of the VBF channel with $\sqrt{s}=13 \mathrm{TeV}$ data [45]. The VBF signal events are required to produce at least two jets with transverse momentum $p_{T}>80(40) \mathrm{GeV}$ for the leading (subleading) jet and rapidity $|\eta|<4.7$. Further, at least one of the two leading jets must have $|\eta|>3$. Events are also required to have a large transverse missing energy, $E_{T}^{\prime}>$ $250 \mathrm{GeV}$.

The dominant SM background contribution to the signal region arises from the $W+$ jets and $Z+$ jets channels, with $W$ and $Z$ decaying leptonically into $\ell \nu_{\ell}$ and $\nu_{\ell} \bar{\nu}_{\ell}$, respectively. The $W+$ jets channel contributes due to a nonzero lepton misidentification probability combined with a large production cross section. The next largest contributions are due to the top production (single and pair) and gauge boson pair production. Contributions from the top production channels are suppressed by the small lepton misidentification probability, whereas the gauge boson pair production channels suffer from smaller cross sections. QCD jets and $\gamma+$ jets are the other two potentially large contributors to the background. Although they do not have any direct source of missing energy in the final state, mismeasurements of the momenta of the final state particles, especially jets, can result in a nonzero $E_{T}$.

In order to suppress the QCD background contribution, all the jets in the final state with $p_{T}>30 \mathrm{GeV}$ and $|\eta|<$ 4.7 are required to be separated from the missing momentum direction $\overrightarrow{E_{T}}$ by an azimuthal angle $\Delta \phi\left(\overrightarrow{E_{T}}, \overrightarrow{p_{T}}{ }^{\text {jet }}\right)>$ 0.5 radians. The two leading jets in the VBF channel are expected to be widely separated by pseudorapidity $\eta$ and almost back to back. This kinematic feature is exploited by requiring $\eta_{j 1} \eta_{j 2}<0$ and $\left|\Delta \eta_{j j}\right|>4$. These jets are also likely to have a large invariant mass, $m_{j j}>1300 \mathrm{GeV}$, and a small azimuthal separation, $\left|\Delta \phi_{j j}\right|<1.5$. Such cuts are particularly effective in reducing the SM background contributions arising from the $V+$ jets channel, where $V=W, Z$. The $W+$ jets background is further reduced by implementing a lepton veto. Events are rejected if they contain a muon (electron) with $p_{T}>10 \mathrm{GeV}$ and $|\eta|<$ $2.4(2.5)$ or a $\tau$-lepton decaying hadronically $\left(\tau_{h}\right)$ with $p_{T}>$ $10 \mathrm{GeV}$ and $|\eta|<2.3$. Any event with a photon in the final state with $p_{T}>15 \mathrm{GeV}$ and $|\eta|<2.5$ is also rejected to suppress the $\gamma+$ jets background. A $b$-jet veto is implemented in order to reduce the background from the $t \bar{t}$ and single top production channels. The $b$-jet candidates
TABLE I. Final state selection cuts for the VBF analysis [45].

\begin{tabular}{lc}
\hline \hline Observables & Requirements \\
\hline Leading (trailing) jet & $p_{T}>80(40) \mathrm{GeV},|\eta|<4.7$ \\
$E_{T}$ & $>250 \mathrm{GeV}$ \\
$\Delta \phi\left(\vec{E}_{T}, \vec{p}_{T}^{\text {jet }}\right)$ & $>0.5$ \\
$\left|\Delta \phi_{j j}\right|$ & $<1.5$ \\
$\eta_{j 1} \cdot \eta_{j 2}$ & $<0$ \\
$\left|\Delta \eta_{j j}\right|$ & $>4$ \\
$\left|m_{j j}\right|$ & $>1300 \mathrm{GeV}$ \\
Leptons & $N_{\mu, e}=0, p_{T}>10 \mathrm{GeV},|\eta|<2.4(2.5)$ \\
$\tau$ leptons & $N_{\tau_{h}}=0, p_{T}>10 \mathrm{GeV},|\eta|<2.3$ \\
Photons & $N_{\gamma}=0, p_{T}>15 \mathrm{GeV},|\eta|<2.5$ \\
$b$-jets & $N_{b}=0, p_{T}>20 \mathrm{GeV},|\eta|<2.4$ \\
\hline \hline
\end{tabular}

are required to have $p_{T}>20 \mathrm{GeV}$ and $|\eta|<2.4$. The combined secondary vertex algorithm $[46,47]$ adopted by the CMS Collaboration yields roughly a $60 \%$ efficiency of tagging the $b$-jets. A small probability (1\%) of misidentifying a light-flavor jet as a $b$-jet is also taken into account. We have summarized the selection criteria for the final state in Table I.

The model has been implemented in MADGRAPH5 $[48,49]$ through FEYNRULES [50-52]. Event generation at the parton level is performed by MADGRAPH. We have used the parton distribution function NNPDF $[53,54]$ for our computation. These events are subsequently passed to PYTHIA8 [55] for showering and hadronization. Jet formation is done using the anti-kT algorithm [56] by FASTJET [57] and detector simulation is performed using DeLPHES3 [58-60]. Finally, the events are analysed by CHECKMATE [61,62].

\section{B. Results and discussion}

In this section, we discuss the results of our collider simulation for two choices of the heavy $C P$-even Higgs mass, $m_{h_{2}}=150$ and $300 \mathrm{GeV}$. Since the $h_{2}$ production is suppressed by small $\sin ^{2} \theta$, we restrict the $h_{2}$ mass to the electroweak range.

In order to estimate the statistical significance factor $\mathcal{S}$, we use

$$
\mathcal{S}=\frac{S}{\sqrt{S+B+\sigma_{B}^{2}}},
$$

where $S, B$ and $\sigma_{B}$ represent the number of signal events, SM background events and the uncertainty in the background measurement. The CMS Collaboration quotes $B \pm \sigma_{B}=1779 \pm 96$ at $35.9 \mathrm{fb}^{-1}$ integrated luminosity [45]. To estimate $\mathcal{S}$ at high integrated luminosity, we have scaled $B$ accordingly and taken two choices of $\sigma_{B}$ to reflect our lack of knowledge of how the realistic uncertainty may evolve. The "best case scenario" corresponds to a negligible background uncertainty, $\sigma_{B} \ll \sqrt{B}$, and the second choice is $\sigma_{B}=\sqrt{B}$ which effectively reduces the significance by $\sqrt{2}$. 

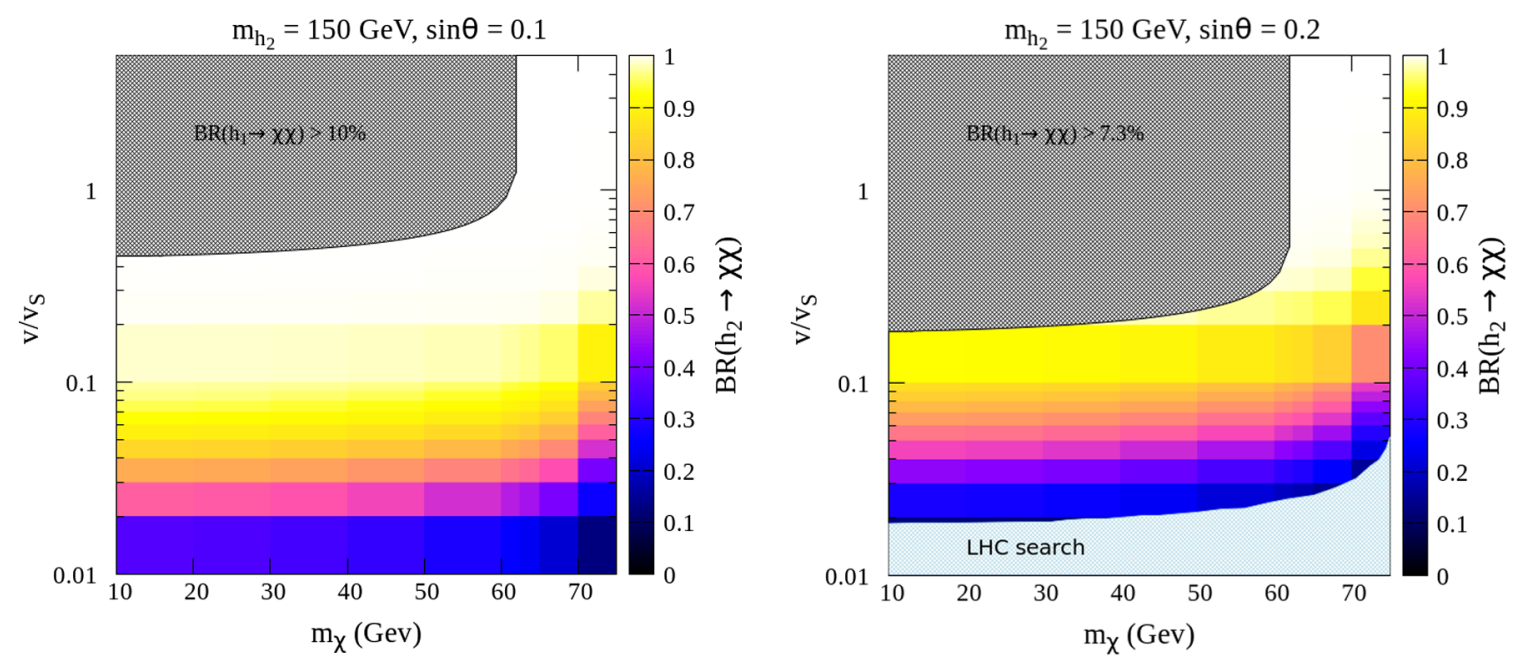

FIG. 7. $\mathrm{BR}\left(h_{2} \rightarrow \chi \chi\right)$ and the LHC constraints for $m_{h_{2}}=150 \mathrm{GeV}$.

We expect that the resulting significance gives an idea of the realistic signal strength and the detection prospects.

Below we consider two representative values of $m_{h_{2}}$ and the resulting signals.

\section{1. $m_{h_{2}}=150 \mathrm{GeV}$}

We fix $\sin \theta$ to be 0.1 and 0.2 since for larger $\sin \theta \geq 0.3$ a $150 \mathrm{GeV} C P$-even Higgs is nearly excluded by the LHC data $[35,37]$. We vary the ratio $v / v_{s}$ within the range [0.01-5.0], where the upper limit is set by the perturbative unitarity constraint [27]. The DM mass $m_{\chi}$ is bounded from above by $m_{h_{2}} / 2$ as required by the on-shell decay of $h_{2}$ into a DM pair.

In Fig. 7, we show the variation of $\operatorname{BR}\left(h_{2} \rightarrow \chi \chi\right)$ in the plane $\left(v / v_{s}, m_{\chi}\right)$ for two choices of $\sin \theta$. As Eq. (2.21) suggests, the branching ratio increases with smaller $\sin \theta$ and $v_{s}$. The upper left corner in both figures is excluded by the invisible decay of the $125 \mathrm{GeV}$ Higgs boson, while the low values of $v / v_{s}$ in the right panel are ruled out by the $h_{2}$ searches. We see that large values of $\mathrm{BR}_{\text {inv }}\left(h_{2}\right)$ close to one are consistent with the LHC constraints.

In Fig. 8, we show the variation of the expected statistical significance of the VBF signal at $13 \mathrm{TeV}$ with an integrated luminosity of $3 \mathrm{ab}^{-1}$ in the $\left(v / v_{s}, m_{\chi}\right)$ plane. The production cross section of $h_{1}$ is much larger than that of $h_{2}$, whereas its coupling to DM is much smaller. The balance between the $h_{1}$ and $h_{2}$ contributions to the event rate depends on the parameter choice. Let us consider a few benchmark points (BP).

(i) $\mathrm{BP} 1\left(\sin \theta=0.2, v / v_{s}=0.05, m_{\chi} \simeq 50 \mathrm{GeV}\right)$. The total signal cross section at this point amounts to $0.26 \mathrm{fb}$ with $\mathrm{BR}\left(h_{1} \rightarrow \chi \chi\right) \simeq 2.9 \times 10^{-3}$ and $\operatorname{BR}\left(h_{2} \rightarrow \chi \chi\right) \simeq 0.54$. This leads to the final state dominated by the $h_{2}$ decay contribution accounting for $90 \%$ of the signal events.

(ii) $\mathrm{BP} 2\left(\sin \theta=0.2, v / v_{s}=0.2, m_{\chi} \simeq 50 \mathrm{GeV}\right)$. The total signal cross section is $0.93 \mathrm{fb}$ with $\mathrm{BR}\left(h_{1} \rightarrow\right.$ $\chi \chi) \simeq 0.045$ and $\operatorname{BR}\left(h_{2} \rightarrow \chi \chi\right) \simeq 0.96$. Here we have almost equal contributions from $h_{1}$ and $h_{2}$ : the on-shell decay of $h_{2}$ accounts for $51 \%$ of the signal events. Note that the smallness of the $h_{2}$ production cross section is duly compensated by its large invisible decay branching ratio and slightly better cut efficiency.

Figure 8 shows that there are good prospects for light $\left(m_{\chi}<60 \mathrm{GeV}\right)$ DM detection as long as the background uncertainty does not substantially exceed $\sqrt{B}$. The signal significance can reach 5 and higher for $v / v_{s}>0.1$. The thermal WIMP band can only be probed in the vicinity of the resonance, i.e., for DM masses close to $m_{h_{1}} / 2$.

We find that since the off-shell $h_{1,2}$ contributions to the cross section are small, there is no hope of probing the DM mass beyond $m_{h_{2}} / 2$.

\section{2. $m_{h_{2}}=300 \mathrm{GeV}$}

Unlike in the previous case, now $h_{2}$ can decay on shell into $W W, Z Z$, and $h_{1} h_{1}$ final states, with the branching ratio for the gauge boson modes being the largest. As a result, one has to lower $\sin \theta$ and/or increase $v / v_{s}$ in order to obtain a large enough $\operatorname{BR}\left(h_{2} \rightarrow \chi \chi\right)$. However, a small $\sin \theta \sim 0.1$ also suppresses the $h_{2}$ production cross section, while a large $v / v_{s}$ would be in conflict with unitarity. Thus, we focus on the mid-range values $\sin \theta=0.2,0.3$. In Fig. 9, we show the resulting $\operatorname{BR}\left(h_{2} \rightarrow \chi \chi\right)$ distribution.

Figure 10 displays the variation of expected statistical significance of the VBF signal at the $13 \mathrm{TeV}$ LHC with an integrated luminosity of $3 \mathrm{ab}^{-1}$. The cut efficiency improves compared to the previous case, while the cross section and $\operatorname{BR}\left(h_{2} \rightarrow \chi \chi\right)$ reduce. The net result is that the expected significance drops, with the maximal value being 4 to 5 at $v / v_{s} \sim 1$.

Let us consider the benchmark points with the same $m_{\chi}$ and $\sin \theta$ as in BP1 and BP2 in order to assess the quantitative changes. 

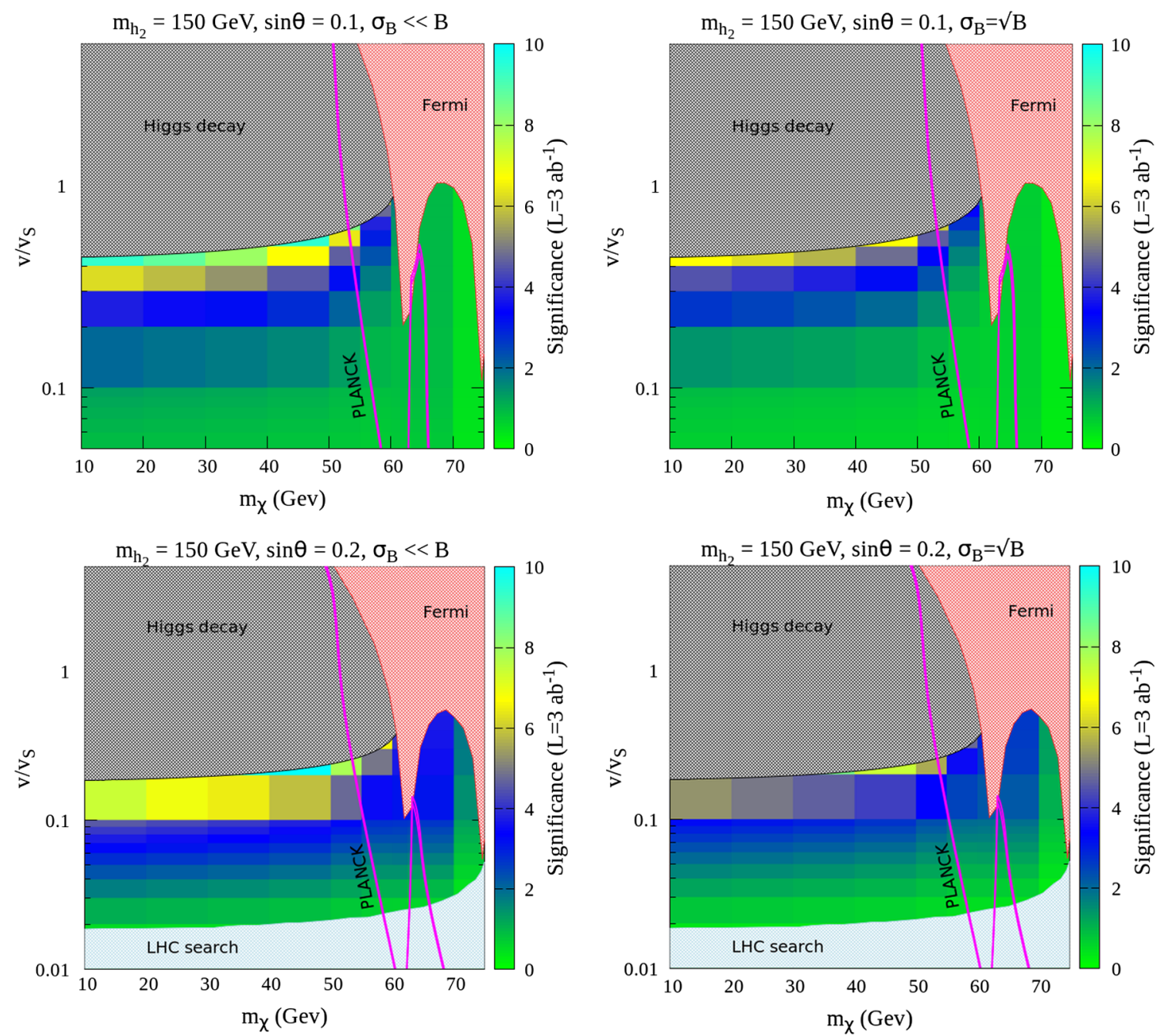

FIG. 8. Significance reach for the VBF channel with missing energy at $L=3 \mathrm{ab}^{-1}$ and $m_{h_{2}}=150 \mathrm{GeV}$. The shaded regions are excluded by the LHC and Fermi observations, while the purple band is preferred by PLANCK assuming thermal DM production. The left and right panels assume different background uncertainties: $\sigma_{B}=0$ and $\sqrt{B}$, respectively.
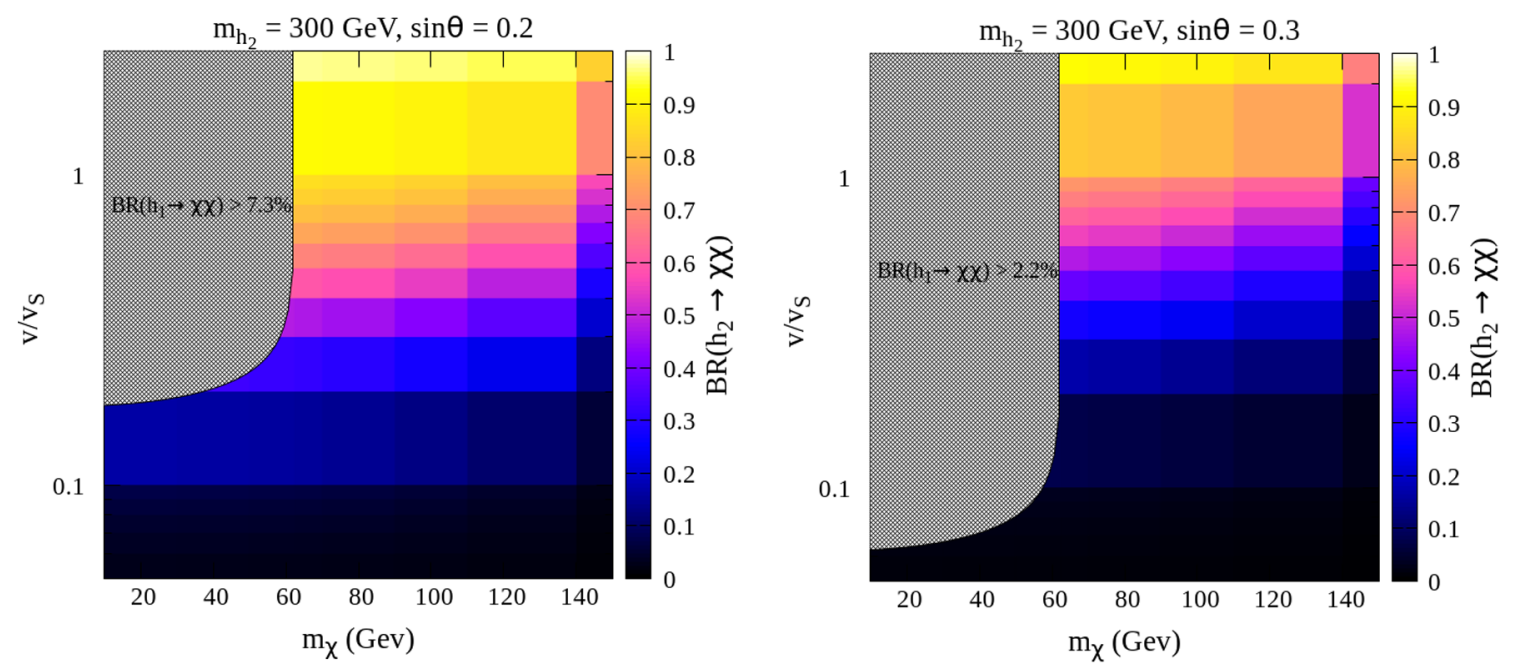

FIG. 9. $\quad \mathrm{BR}\left(h_{2} \rightarrow \chi \chi\right)$ and the LHC constraints for $m_{h_{2}}=300 \mathrm{GeV}$. 

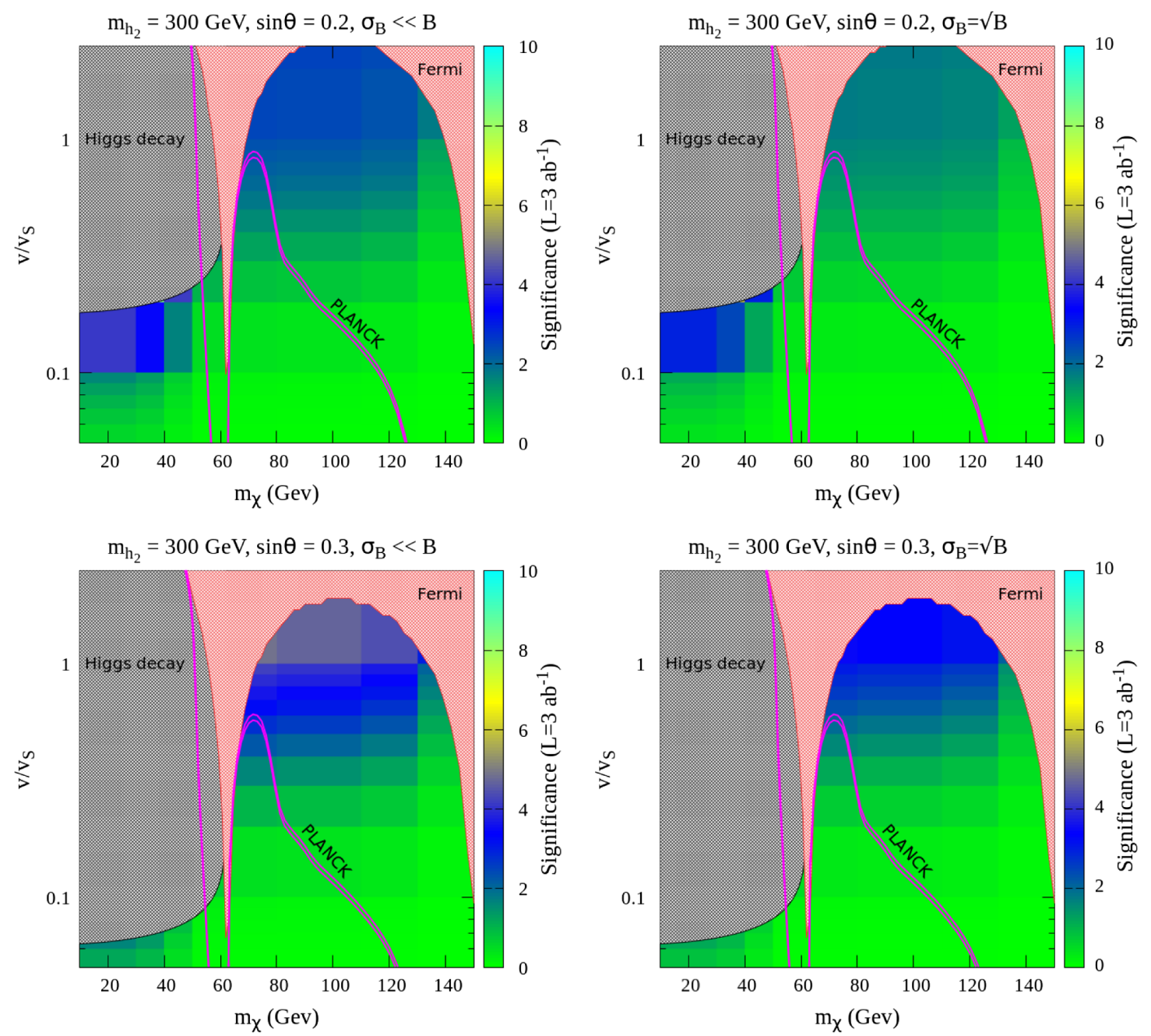

FIG. 10. Significance reach for the VBF channel with missing energy at $L=3 \mathrm{ab}^{-1}$ and $m_{h_{2}}=300 \mathrm{GeV}$. The shaded regions are excluded by the LHC and Fermi observations, while the purple band is preferred by PLANCK assuming thermal DM production. The left and right panels assume different background uncertainties: $\sigma_{B}=0$ and $\sqrt{B}$, respectively.

(i) $\mathrm{BP} 3\left(\sin \theta=0.2, v / v_{s}=0.05, m_{\chi} \simeq 50 \mathrm{GeV}\right)$. The total signal cross section is $0.04 \mathrm{fb}$ with $\mathrm{BR}\left(h_{1} \rightarrow\right.$ $\chi \chi) \simeq 2.9 \times 10^{-3}$ and $\mathrm{BR}\left(h_{2} \rightarrow \chi \chi\right) \simeq 0.02$. About $35 \%$ of the signal events arise from the on-shell decay of $h_{2}$.

(ii) $\mathrm{BP} 4\left(\sin \theta=0.2, v / v_{s}=0.2, m_{\chi} \simeq 50 \mathrm{GeV}\right)$. The total signal cross section is $0.57 \mathrm{fb}$ with $\mathrm{BR}\left(h_{1} \rightarrow\right.$ $\chi \chi) \simeq 0.05$ and $\operatorname{BR}\left(h_{2} \rightarrow \chi \chi\right) \simeq 0.24$. The on-shell decay of $h_{2}$ accounts for $31 \%$ of the signal events. Evidently, the signal rate is weaker for a heavier $h_{2}$. On the other hand, one can probe somewhat heavier DM up to about $120 \mathrm{GeV}$ and in certain (rather limited) regions the signal significance reaches the discovery threshold. As before, the thermal WIMP can only be probed in the vicinity of the resonance, i.e., for $m_{\chi} \sim m_{h_{1}} / 2$.

Let us close this section with a note. The CMS Collaboration has recently published its projected reach for the invisible decay of the $125 \mathrm{GeV}$ Higgs at high luminosity LHC. The $95 \%$ C.L. upper bound on $\mathrm{BR}_{\mathrm{inv}}$ assuming SM-like production of the Higgs boson is expected to be $3.8 \%$ with $3 \mathrm{ab}^{-1}$ integrated luminosity at $14 \mathrm{TeV}$ [63]. They have optimized the sensitivity using the cuts $E_{T}>190 \mathrm{GeV}$ and $\left|m_{j j}\right|>2500 \mathrm{GeV}$ for this analysis. We have checked that these cuts coupled with the ones in Table I improve the significance factor in our scenario only slightly.

\section{SUMMARY AND CONCLUSIONS}

We have studied the dark matter discovery prospects in the Higgs portal framework with pseudo-Goldstone DM. The model is particularly attractive due to its simplicity and elegant cancellation of the direct detection amplitude, which allows for a wide range of DM masses consistent with XENON1T. We have focused on the VBF production of the Higgs-like scalars which decay into DM pairs, thereby producing the "missing $E_{T}$ " signature. Taking into account the current LHC bounds along with the indirect 
DM detection constraint from Fermi, we find that relatively light, $m_{\chi} \lesssim 100 \mathrm{GeV}$, dark matter can be probed in this channel with the signal significance at $L=3 \mathrm{ab}^{-1}$ reaching the discovery threshold in certain regions of parameter space.

The model predicts the existence of a heavier Higgs-like boson $h_{2}$ with suppressed couplings. This would provide a complementary test of the model, although its detection is hindered by the strong mixing angle suppression and a large invisible decay width. It is noteworthy that $h_{2}$ couples to dark matter much stronger than the SM-like Higgs $h_{1}$ does, hence its invisible decay can be very efficient even though the invisible decay of $h_{1}$ is severely constrained.

In this scenario, dark matter can be light quite naturally since its mass is provided by a symmetry breaking term.
The direct detection constraints are very weak, so the lower bound of the order of a few $\mathrm{GeV}$ is only set by the $B$-meson decays. Although we focus on the DM mass range above $10 \mathrm{GeV}$, essentially all of our results apply to lower masses as well.

\section{ACKNOWLEDGMENTS}

O. L. acknowledges support from the Academy of Finland project "The Higgs boson and the Cosmos." K. H., N. K., and S.M. acknowledge H2020-MSCARISE-2014 Grant No. 645722 (nonminimal Higgs). T. T. is partially supported by the Grant-in-Aid for Scientific Research on Innovative Areas from the MEXT (PI: Koji Tsumura, Grant No. 18H05543).
[1] E. Aprile et al. (XENON Collaboration), Phys. Rev. Lett. 121, 111302 (2018).

[2] X. Cui et al. (PandaX-II Collaboration), Phys. Rev. Lett. 119, 181302 (2017).

[3] C. Gross, O. Lebedev, and T. Toma, Phys. Rev. Lett. 119, 191801 (2017).

[4] M. Ibe, Y. Nakayama, H. Murayama, and T. T. Yanagida, J. High Energy Phys. 04 (2009) 087.

[5] M. Frigerio, T. Hambye, and E. Masso, Phys. Rev. X 1, 021026 (2011).

[6] M. Frigerio, A. Pomarol, F. Riva, and A. Urbano, J. High Energy Phys. 07 (2012) 015.

[7] J. Jaeckel, Phys. Lett. B 732, 1 (2014).

[8] T. Alanne, H. Gertov, F. Sannino, and K. Tuominen, Phys. Rev. D 91, 095021 (2015).

[9] Y. Mambrini and T. Toma, Eur. Phys. J. C 75, 570 (2015).

[10] A. Carmona and M. Chala, J. High Energy Phys. 06 (2015) 105.

[11] D. Barducci, A. Bharucha, N. Desai, M. Frigerio, B. Fuks, A. Goudelis, S. Kulkarni, G. Polesello, and D. Sengupta, J. High Energy Phys. 01 (2017) 078.

[12] G. Ballesteros, A. Carmona, and M. Chala, Eur. Phys. J. C 77, 468 (2017).

[13] R. Balkin, M. Ruhdorfer, E. Salvioni, and A. Weiler, J. High Energy Phys. 11 (2017) 094.

[14] R. Balkin, M. Ruhdorfer, E. Salvioni, and A. Weiler, J. Cosmol. Astropart. Phys. 11 (2018) 050.

[15] A. Djouadi, O. Lebedev, Y. Mambrini, and J. Quevillon, Phys. Lett. B 709, 65 (2012).

[16] J. McDonald, Phys. Rev. D 50, 3637 (1994).

[17] V. Barger, P. Langacker, M. McCaskey, M. Ramsey-Musolf, and G. Shaughnessy, Phys. Rev. D 79, 015018 (2009).

[18] E. Gabrielli, M. Heikinheimo, K. Kannike, A. Racioppi, M. Raidal, and C. Spethmann, Phys. Rev. D 89, 015017 (2014).

[19] M. Gonderinger, H. Lim, and M. J. Ramsey-Musolf, Phys. Rev. D 86, 043511 (2012).
[20] B. Grzadkowski and D. Huang, J. High Energy Phys. 08 (2018) 135.

[21] D. Azevedo, M. Duch, B. Grzadkowski, D. Huang, M. Iglicki, and R. Santos, J. High Energy Phys. 01 (2019) 138.

[22] K. Ishiwata and T. Toma, J. High Energy Phys. 12 (2018) 089.

[23] G. Aad et al. (ATLAS and CMS Collaborations), J. High Energy Phys. 08 (2016) 045.

[24] A. M. Sirunyan et al. (CMS Collaboration), Eur. Phys. J. C 79, 421 (2019).

[25] A. Falkowski, C. Gross, and O. Lebedev, J. High Energy Phys. 05 (2015) 057.

[26] G. Belanger, F. Boudjema, A. Pukhov, and A. Semenov, Comput. Phys. Commun. 185, 960 (2014).

[27] C.-Y. Chen, S. Dawson, and I. M. Lewis, Phys. Rev. D 91, 035015 (2015).

[28] M. Ackermann et al. (Fermi-LAT Collaboration), Phys. Rev. Lett. 115, 231301 (2015).

[29] A. Albert et al. (Fermi-LAT and DES Collaborations), Astrophys. J. 834, 110 (2017).

[30] Combined measurements of Higgs boson production and decay using up to $80 \mathrm{fb}^{-1}$ of proton-proton collision data at $\sqrt{s}=13 \mathrm{TeV}$ collected with the ATLAS experiment, CERN Technical Report No. ATLAS-CONF-2018-031, 2018, https://atlas.web.cern.ch/Atlas/GROUPS/PHYSICS/ CONFNOTES/ATLAS-CONF-2018-031/.

[31] J. F. Navarro, C. S. Frenk, and S. D. M. White, Astrophys. J. 490, 493 (1997).

[32] P. A. R. Ade et al. (Planck Collaboration), Astron. Astrophys. 594, A13 (2016).

[33] M. Aaboud et al. (ATLAS Collaboration), Phys. Lett. B 775, 105 (2017).

[34] A. M. Sirunyan et al. (CMS Collaboration), Phys. Rev. D 98, 092001 (2018).

[35] M. Aaboud et al. (ATLAS Collaboration), Eur. Phys. J. C 78, 24 (2018).

[36] M. Aaboud et al. (ATLAS Collaboration), Phys. Rev. D 98, 052008 (2018). 
[37] M. Aaboud et al. (ATLAS Collaboration), Eur. Phys. J. C 78, 293 (2018).

[38] A. M. Sirunyan et al. (CMS Collaboration), J. High Energy Phys. 06 (2018) 127.

[39] M. Aaboud et al. (ATLAS Collaboration), J. High Energy Phys. 01 (2019) 030.

[40] M. Aaboud et al. (ATLAS Collaboration), J. High Energy Phys. 11 (2018) 040.

[41] A. M. Sirunyan et al. (CMS Collaboration), Phys. Lett. B 788, 7 (2019).

[42] A. M. Sirunyan et al. (CMS Collaboration), J. High Energy Phys. 08 (2018) 152.

[43] M. Aaboud et al. (ATLAS Collaboration), Phys. Lett. B 793, 499 (2019).

[44] J. S. Kim, O. Lebedev, and D. Schmeier, J. High Energy Phys. 11 (2015) 128.

[45] A. M. Sirunyan et al. (CMS Collaboration), Phys. Lett. B 793, 520 (2019).

[46] S. Chatrchyan et al. (CMS Collaboration), J. Instrum. 8, P04013 (2013).

[47] A. M. Sirunyan et al. (CMS Collaboration), J. Instrum. 13, P05011 (2018).

[48] J. Alwall, M. Herquet, F. Maltoni, O. Mattelaer, and T. Stelzer, J. High Energy Phys. 06 (2011) 128.

[49] J. Alwall, R. Frederix, S. Frixione, V. Hirschi, F. Maltoni, O. Mattelaer, H. S. Shao, T. Stelzer, P. Torrielli, and M. Zaro, J. High Energy Phys. 07 (2014) 079.

[50] N. D. Christensen and C. Duhr, Comput. Phys. Commun. 180, 1614 (2009).
[51] A. Alloul, N. D. Christensen, C. Degrande, C. Duhr, and B. Fuks, Comput. Phys. Commun. 185, 2250 (2014).

[52] C. Degrande, C. Duhr, B. Fuks, D. Grellscheid, O. Mattelaer, and T. Reiter, Comput. Phys. Commun. 183, 1201 (2012).

[53] R. D. Ball et al., Nucl. Phys. B867, 244 (2013).

[54] R. D. Ball et al. (NNPDF Collaboration), J. High Energy Phys. 04 (2015) 040.

[55] T. Sjöstrand, S. Ask, J. R. Christiansen, R. Corke, N. Desai, P. Ilten, S. Mrenna, S. Prestel, C. O. Rasmussen, and P. Z. Skands, Comput. Phys. Commun. 191, 159 (2015).

[56] M. Cacciari, G. P. Salam, and G. Soyez, J. High Energy Phys. 04 (2008) 063.

[57] M. Cacciari, G. P. Salam, and G. Soyez, Eur. Phys. J. C 72, 1896 (2012).

[58] J. de Favereau, C. Delaere, P. Demin, A. Giammanco, V. Lemaître, A. Mertens, and M. Selvaggi (DELPHES 3 Collaboration), J. High Energy Phys. 02 (2014) 057.

[59] M. Selvaggi, J. Phys. Conf. Ser. 523, 012033 (2014).

[60] A. Mertens, J. Phys. Conf. Ser. 608, 012045 (2015).

[61] M. Drees, H. Dreiner, D. Schmeier, J. Tattersall, and J. S. Kim, Comput. Phys. Commun. 187, 227 (2015).

[62] D. Dercks, N. Desai, J. S. Kim, K. Rolbiecki, J. Tattersall, and T. Weber, Comput. Phys. Commun. 221, 383 (2017).

[63] Search for invisible decays of a Higgs boson produced through vector boson fusion at the High-Luminosity LHC, CERN Technical Report No. CMS-PAS-FTR-18016, 2018. 